\title{
Survival in Cirrhotic Patients with High MELD Scores: The TIPping Point
}

\author{
Gloria L. Hwang ${ }^{1}$ Daniel Y. Sze ${ }^{1}$
}

Published online: 9 November 2016

(c) Springer Science+Business Media New York 2016

\begin{abstract}
Although a survival benefit following transjugular intrahepatic portosystemic shunt (TIPS) creation for variceal bleeding [1] and ascites [2, 3] has been reported in randomized controlled trials (RCTs) in cirrhotic patients, clinical outcomes after TIPS placement in patients with late-stage liver disease and high Model for End-Stage Liver Disease (MELD) scores remain controversial. The MELD score was originally created to predict the survival after TIPS placement in patients with cirrhosis; by intention, a high MELD score predicts poor survival after TIPS [4]. While the RCTs supported early TIPS placement for variceal bleeding and favored TIPS over repeated large-volume paracentesis in patients with Child-Pugh scores up to 11 or 13 , the investigators intentionally excluded patients with severely compromised liver function and higher scores. The RCTs also did not report on outcomes relative to the MELD scores.

Consequently, data regarding TIPS outcomes in patients with high MELD scores have historically been derived from non-randomized studies. Angermayr et al. [5] reported a 60\% 1-year mortality rate in patients with MELD $>18$. Ferral et al. [6] reported a 90-day mortality rate of $34.8 \%$ in patients after elective TIPS with MELD scores of $18-24$ and $65.5 \%$ in patients with MELD $\geq 18$, yielding a 90-day mortality of $44 \%$ for all patients with MELD $\geq 18$. Casadaban et al. [7] stated that survival rates after emergent TIPS placement for acute variceal bleeding were predicted by MELD scores and were inferior to
\end{abstract}

Daniel Y. Sze

dansze@stanford.edu

1 Division of Interventional Radiology, Stanford University School of Medicine, H-3646 Stanford University Medical Center, Stanford, CA 94305-5642, USA survival rates reported in the elective TIPS placement population, with a 90-day mortality rate of $36 \%$ for MELD $19-25$ and $83 \%$ for MELD $\geq 26$.

The high post-TIPS mortality rates reported in patients with high MELD scores have led some to conclude that TIPS procedures should not be performed in those patients, particularly if the MELD is $>25$ [6]. Although such a conclusion implies that TIPS placement is harmful, or at least, not beneficial to that subset of patients, this assumption remains conjectural. The prognosis of patients with high MELD scores is generally poor, with reported 1-year mortality of approximately $30 \%$ for MELD scores between 20 and 29 and approximately $60 \%$ for MELD scores $\geq 30$ [8]. Given the variability among patient cohorts in how MELD scores are stratified, and in how survival outcomes are reported, and given the similarities in the reported survival statistics for high-MELD patients with and without TIPS, it is difficult to isolate the effect of TIPS placement from the overall dismal survival typical of highMELD patients.

In this issue of Digestive Diseases and Sciences, Ascha et al. [9] have taken a step toward answering the question of whether TIPS placement increases or decreases survival in patients with high MELD scores. They retrospectively analyzed a cohort of 144 patients with MELD score $\geq 15$ who underwent TIPS procedures, matched them by MELD score and age to 144 patients who had not undergone TIPS procedures, excluding patients with hepatocellular carcinoma or those who died within $48 \mathrm{~h}$ of the TIPS procedure. They described that in the first 2 months, TIPS patients had a 56\% higher risk for dying or requiring orthotopic liver transplantation (OLT) than matched non-TIPS patients, which was statistically significant when corrected for Child-Pugh score. After the first 2 months, though, TIPS patients had a $47 \%$ lower risk of dying or requiring OLT. 
The 1-year transplant-free survival in both groups was low (29\% in TIPS patients, $19 \%$ in non-TIPS patients).

At first glance, these survival numbers are substantially inferior to those previously reported in the literature. What could be the source of this disparity? One explanation is a methodological differences between the current study and prior studies $[2-4,8]$. Other studies censored patients who underwent OLT, such that the date of transplantation was considered the last day of follow-up and not counted as an event. In the current study, both death and OLT are reported as events that count against transplant-free survival. The authors report that $55 \%$ of subjects died during this period and $27 \%$ underwent liver transplantation. A $55 \%$ mortality rate for the study period is better aligned with the reported survival results of other studies.

Because OLT and death have been combined as a single outcome, it is difficult to determine the actual mortality risk of TIPS from the data presented. As Malinchoc et al. had noted in their study, censoring on the day of OLT avoids the influence of deaths due to surgical mortality and also avoids the influence of OLT in prolonging survival, since survival in patients who undergo OLT is significantly improved compared with those who do not undergo OLT [4]. In the current study, by treating OLT and death as the same outcome, the authors imply that OLT is a negative outcome, when in fact it is likely to improve patient survival. Although it is unclear how many of the patients in each cohort underwent OLT, the second most common reason not to pursue TIPS in the non-TIPS cohort was ineligibility for liver transplantation, since TIPS is a recognized tool to bridge patients to transplantation. Another potential issue in this study is, as the authors acknowledge, selection bias between the TIPS and non-TIPS cohorts (Table 1). The authors matched the patients according to age and MELD score, two excellent predictors of survival outcome in cirrhosis $[8,10]$. Nonetheless, this matching algorithm does not take into account other factors that may affect baseline prognosis. By definition, all patients undergoing TIPS have experienced a complication of endstage liver disease (ESLD), since the indications for TIPS are in fact such complications including variceal bleeding and refractory ascites. It is unclear whether any or all of the non-TIPS patients experienced similar complications. It is likely that a greater percentage of the TIPS cohort had a history of gastrointestinal bleeding, since bleeding was the cause of death in 25 TIPS patients versus eight non-TIPS patients. As D'Amico et al. [10] described, patients who experience gastrointestinal bleeding with or without ascites have a substantially poorer prognosis, with a $57 \%$ mortality rate/year, as compared to a $20 \%$ mortality rate/year for patients with ascites without variceal bleeding. If the TIPS cohort had a higher percentage of patients who had experienced gastrointestinal bleeding, the prognosis for that cohort would be expected to be inferior at baseline. Furthermore, Said et al. [8] demonstrated that patients with any complications of cirrhosis, including those other than variceal bleeding such as encephalopathy and ascites, had significantly higher 1-year mortality rates than those with compensated cirrhosis even when matched by MELD scores. In the current study, $100 \%$ of the TIPS cohort had decompensated cirrhosis, but the percentage in the nonTIPS cohort was not reported and was probably lower, again suggesting that the pre-intervention prognosis for the TIPS patients may have been inferior to that of the nonTIPS cohort. Of note, patients who required emergent TIPS were not excluded from the current study unless they died within $48 \mathrm{~h}$, further biasing the cohort toward a graver baseline prognosis [7].

Given the many reasons that the cohorts were not perfectly matched and that the TIPS cohort may have had an unfavorable baseline prognosis when compared with ageand MELD-matched non-TIPS patients, the suggestion of superior survival in the TIPS cohort beyond 2 months is remarkable. The lack of significant difference in the

Table 1 Potential mismatching between cohorts

\begin{tabular}{lccl}
\hline & $\begin{array}{l}\text { TIPS } \\
\text { cohort }\end{array}$ & Non-TIPS cohort & Expected effect \\
\hline Patient desires TIPS & Yes & $\begin{array}{c}\text { Probably not (\#1 most } \\
\text { common reason for not } \\
\text { receiving TIPS) }\end{array}$ & - \\
Transplant eligible & Probably & $\begin{array}{c}\text { Possibly not (\#2 most } \\
\text { common reason for not } \\
\text { receiving TIPS) }\end{array}$ & $\begin{array}{c}\text { More transplants in TIPS patients }=\text { more } \\
\text { nonlethal "events" degrading survival } \\
\text { curve }\end{array}$ \\
$\begin{array}{l}\text { Decompensation (refractory acute or chronic } \\
\text { hemorrhage, refractory ascites requiring repeated } \\
\text { large-volume paracentesis) }\end{array}$ & $\begin{array}{c}\text { Yes- } \\
100 \%\end{array}$ & $\begin{array}{c}\text { Not reported-100\% had } \\
\text { ascites, but severity of } \\
\text { ascites not specified }\end{array}$ & $\begin{array}{c}\text { Decompensation, especially acute } \\
\text { hemorrhage, is associated with } \\
\text { significantly worse baseline prognosis }\end{array}$ \\
\hline
\end{tabular}


incidence of hepatic encephalopathy between the two cohorts is also remarkable in that it challenges the common belief that TIPS placement causes encephalopathy, particularly in the high MELD population.

The authors have shed much-needed light on survival and encephalopathy outcomes in these high-risk patients. While a RCT would provide more definitive data on the contribution of TIPS to survival in high-MELD patients, it would be impossible to persuade exsanguinating patients to agree to the risk of being randomized to a non-TIPS arm. Using the existing cohorts, it would still be of interest to determine whether matching not only for MELD and age but also for decompensating events, using death and not OLT as the survival endpoint, would influence the outcomes, especially the short-term results. For this high MELD score patient population with limited therapeutic options, what is suggested in this study is tantalizing: that TIPS may not only be a safe treatment option but may actually be beneficial to survival.

\section{References}

1. García-Pagán JC, Caca K, Bureau C, et al. Early use of TIPS in patients with cirrhosis and variceal bleeding. $N$ Engl $\mathrm{J} \mathrm{Med.}$ 2010;362:2370-2379.
2. Rössle M, Ochs A, Gülberg V, et al. Comparison of paracentesis and transjugular intrahepatic portosystemic shunting in patients with ascites. N Engl J Med. 2000;342:1701-1707.

3. Salerno F, Merli M, Riggio O, et al. Randomized controlled study of TIPS versus paracentesis plus albumin in cirrhosis with severe ascites. Hepatology. 2004;40:629-635.

4. Malinchoc M, Kamath PS, Gordon FD, Peine CJ, Rank J, Terborg PC. A model to predict poor survival in patients undergoing transjugular intrahepatic portosystemic shunts. Hepatology. 2000;31:864-871.

5. Angermayr B, Cejna M, Karnel F, et al. Child-Pugh versus MELD score in predicting survival in patients undergoing transjugular intrahepatic portosystemic shunt. Gut. 2003;52:879-885.

6. Ferral H, Gamboa P, Postoak DW, et al. Survival after elective transjugular intrahepatic portosystemic shunt creation: prediction with model for end-stage liver disease score. Radiology. 2004;231:231-236.

7. Casadaban LC, Parvinian A, Zivin SP, et al. MELD score for prediction of survival after emergent TIPS for acute variceal hemorrhage: derivation and validation in a 101-patient cohort. Ann Hepatol. 2015;14:380-388.

8. Said A, Williams J, Holden J, et al. Model for end stage liver disease score predicts mortality across a broad spectrum of liver disease. J Hepatol. 2004;40:897-903.

9. Ascha M, Hanouneh M, Ascha MS, et al. Transjugular intrahepatic porto-systemic shunt in patients with liver cirrhosis and model for end-stage liver disease $\geq 15$. Dig Dis Sci. (Epub ahead of print). doi:10.1007/s10620-016-4185-3.

10. D'Amico GD, Garcia-Tsao G, Pagliaro L. Natural history and prognostic indicators of survival in cirrhosis: a systematic review of 118 studies. J Hepatol. 2006;44:217-231. 